\title{
VOCÁBULOS ANTIGOS NAS CRÓNICAS EM PORTUGUÊS DOS PMH (VOL. SCRIPTORES)
}

O volume Scriptores, publicado em 1856 sob a direcção de A. Herculano, e que faz parte dos Portugaliae Monumenta Historica, editados pela Academia das Ciências de Lisboa, é o único que contém crónicas, quer em latim quer em português, geralmente designadas por cronicões. São as seguintes as redigidas em português:

1) Chronica breve do Archivo Nacional [CAN], da 1429 (pgs. 22-23);

2) Chronicas breves e memorias avulsas de S.Cruz de Coimbra [CB1 a 4], da segunda metade do século XV, segundo Herculano, mas duas das quais, com mais verosimilhança, do século XIV, de acordo com Rodrigues Lapa, Lições, p. 266;

3) Vida de D.Tello e noticia da fundação do mosteiro de S.Cruz de Coimbra [VT], de 1455 (pgs. 75-79, versão livre por Álvaro da Mota da Vita Tellonis Archidiaconi notitiaque fundationis coenobii S. Crucis Conimbricensis, inserta em Scriptores (pgs. 64-75);

4) Chronica da fundação do moesteiro de São Vicente de Lixboa pello Inuictissimo e Christiannissimo Dom Afonso Henriquez, $I .{ }^{\circ}$ rei de Portugal: E como tomou a dita çidade aos Mouros [SV], apógrafo transcrito no século XVI sobre texto talvez do século XIV (pgs. 407-414), paráfrase do Indiculum fundationis monasterii S.Vincentii, publicado em Scriptores (pgs. 90-93);

5) Chronica da Conquista do Algarve [CCA] (Chroniqua de como Dom Payo Correa Mestre de Santiago de Castella tomou este reino do Algarve aos Moros), composta talvez no séc. XV, anterior de certeza a 1520 (pgs. 415-420).

Além das siglas referentes às crónicas, entre colchetes, os números escritos em primeiro lugar, no fim dos passos abonatórios (ou depois dos próprios vocábulos), indicam as páginas do vol. Scriptores, onde se encontram os vocábulos ou locuções registados; em segundo lugar designam-se as colunas $1^{\text {a }}$ ou $2^{a}$ pelas letras A e B, respectivamente; em terceiro e último lugar indicam-se as linhas, que são contadas, em todos os casos, desde o alto da página. Todas estas indicações são dadas entre parênteses. Os passos abonatórios, quando apresentam divergência do texto dos Scriptores, reproduzem a nossa leitura, que reputamos exacta, dos manuscritos lá publicados (ou doutros mais antigos), e que, conforme os casos, damos ou daremos à luz (crón. 1 a 4), ou restituímos (crón. 5). O vocábulo ou locução da que se trata vai sublinhado na respectiva citação, para maior clareza. 
Incluímos neste estudo, com a menção de antigas, as palavras arcaicas que sobreviveram ao periodo arcaico da língua ou que revivem literariamente na linguagem dos autores modernos ou contemporâneos e as que designam instituições, costumes ou objectos materiais. Consideramos que o idioma português moderno é o período linguístico que decorre do séc. XVI até à época actual. Por convençăo de utilidade prática, o seu terminus a quo é o ano de 1500 .

Tratamos primeiro dos nomes próprios antigos, especialmente antropónimos e etnónimos:

A la nqu e r, top. ant. (< iuncariu-, através do moçárabe, cf. A.N., it. DE, II, s.v. Alenquer): Alenquer.

"alanquer e ssjntra" (29, A, 45) [CB3]; "alamquer \% Obidos" (25, B, 6) [CB2].

A n riqu e, antr. (m.) ant. ( < germ. latinizado em A genricus, talvez por intermédio do provençal Anric, para poder explicar-se a terminação -ique; significa "senhor" ou "possuidor" (-rik) de cerca (haen) ou de casa (hein); cf. A.N., DE, II, s.v. Henrique): Henrique.

"dom anrique" (24, B, 75) [CB1]; "dom affomso anrrique" $(29, \mathrm{~B}, 20)$ [CB4].

A n ri qu e s, antr. ant., patronímico de Anrique (v.); cf. L.V., Liç. Fil., p. 175 e segs.: Henriques.

"afomso anriquez" (24, B, 16) [CB1]; “dom Afonso anriquez" (407, B, 41, no título do cap. $1^{\circ}$ ) [SV].

Crasto Ma rim, top. ant. (< castru-, seguido do nome da tribo berbere dos Banu Marini ou Merini): Castro Marim.

"no monte (d)onde (era e) hora he Crasto Marjm" (417, A, 48) [CCA].

Crasto Verde, top. ant. $(<$ castru- viride-): Castro Verde. "crasto verde" $(24, \mathrm{~A}, 66)[\mathrm{CB} 1]$.

De $\mathrm{n}$ is, ant. e pop. (nome que veio de França: Saint Denis, apóstolo da Gália, Dionysius, greco-lat. derivado de $\triangle \iota$ ovuoos "Baco"; cf. L.V., Op., III, p. 94, e Antr., p. 46): Dinis.

“D. Denjs“ (416, A, 10) [CCA].

Framengos, etnónimo m. pl. ant. ( $<$ germ. flaming; cf. DP, s.v. flamengo): Flamengos.

"framengos Edoutras naçõẽẽs" (29, A, 33) [CB3].

F r a ndes, top. f. ant. ( $<$ flamengo Vlaanderen; cf. A.N., DE, II, s.v. Flandres): Flandres.

"Oconde philipe de frandes" $(22, \mathrm{~A}, 49)$ [CAN]. 
I ng r a terra, top. f. ant. ( $<$ Angla Terra; cf. A.N., DE, II, s.v. Inglattera): Inglaterra.

"Reys de Jngraterra" (25, B, 32) [CB2].

J es u, hierónimo m. ant. (< Iesu-, do gr. 'Inooũs , do hebr. Iexu; cf. A.N., $D E$, II, s.v. Jesus): Jesus.

A deslocação do acento tónico deve-se à influência da forma grega.

"Jesu christo" $(22, \mathrm{~A}, 20)$ [CAN].

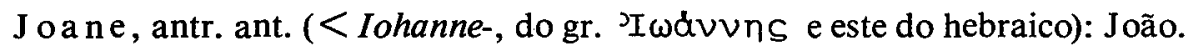
"ho arçebispo Johanne“ $(78, \mathrm{~B}, 50)$ [VT].

Li i nor, antr. f. ant. (< árabe ellinor "o Senhor é a minha luz"; cf. A.N., $D E$, II, s.v. Leonor): Leonor.

"dona lianor" $(22, \mathrm{~B}, 40)$ [CAN].

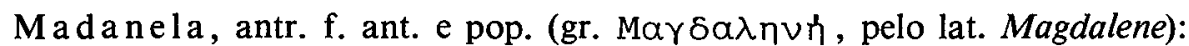
Madalena.

"mujtas lagrimas asy como a mádanela" $(77, \mathrm{~B}, 12)[\mathrm{VT}]$.

M a f omede, antr. m. ant. (< ár. muhammad, através da forma andaluza *Mahumméd; cf. J.P.M., Infl. Ar., II, p. 158): Mafamede.

"seruos de mafomede" (407, A, 28) [SV].

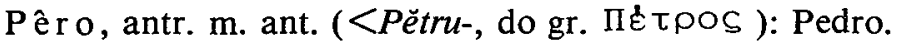
"dom pero afonso" $(28, \mathrm{~B}, 70)$ [CB3].

R omãos, etn. m. pl. ant. (<Rōmānōs): Romanos.

$O$ copista não indicou por lapso a nasalidade.

"uos os romaaos" (28, A, 58) [CB3].

Seguem-se palavras do vocabulário comum existentes, com a mesma grafia, no português moderno ou contemporâneo, mas consideradas antigas por terem também outro sentido além do actual.

a onde $(<a d+$ unde), ant. e pop. no sentido de onde:

"daly a onde estauom" $(417, \mathrm{~A}, 12)$ [CCA].

a u to $(<a c t u-)$ :

Ant. na cepção de acto, como aqui.

"nos autos dos apostollos" (78, A, 53) [VT]. 
c omo (< quōmŏdő): conjunção temporal ant.: quando.

"como tomou leirea aos mouros logo dotou aauera cruz leirea" $(24, \mathrm{~A}, 72)$ [CB1].

c om prid o, ant. no sentido de dotado (de comprir < complere):

"conprido de mujta onestidade" $(75, \mathrm{~B}, 38)$ [VT].

desvair ado, ant. no sentido de vário (particípio de desvairar): "muy desuairadas armas" (411, A, 44) [SV].

d es v a i r o, ant. no sentido de discordância (de desvairar, por desvariar $<$ des + variar < variāre):

“em gramde desvayro (hũs com outros)" $(416, \mathrm{~A}, 52)$ [CCA].

es tilo, ant. no sentido de pena, caneta $(<$ stïlu-):

"tomou huũ estillo de buxo" $(77, \mathrm{~B}, 3)$ [VT].

fazenda ( $<$ facienda): ant. no sentido de: 1. coisas para fazer; 2. peleja, combate:

1. "guisou ssua ffazenda $\mathrm{E}$ ajuntou ssua hoste" (29, A, 28) [CB3]; 2. "Este conde ouue mujtas fazendas cõ mouros. Ecom leoneses" $(29, \mathrm{~B}, 13)$ [CB4]; "veerom aa fazenda em gimarees“ $(29, \mathrm{~B}, 66)$ [CB4].

fim, f., ant. e popular $(<$ fine-): fim, $\mathrm{m}$.

"ataa a sua fim" (77, A, 41) [VT]; "fin(s) do(s) seus dias" (417, A, 26) [CCA]. lhes.

lhe ( $<$ illī em próclise com pal. começadas por vog., como illu-), ant. e pop.:

"aos conçelhes [sic] ffazelhe honrra" (26, A, 51) [CB31.

n oj o (de nojo, der. de enojar < lat. vulg. inŏdiăre, talvez através do prov. ant.; sent. ant. 1. prejuízo; 2 . aborrecimento, desgosto.

1. "tanto dapno e nojo" (416, B, 29) [CCA]; 2. "E quando o bispo E cabidoo virom que dõ tello queria ordenar moesteiro tomarom muj grande nojo" $(76, \mathrm{~B}, 15)$ [VT].

nova me nte, ant. no sent. de: recentemente, pouco antes.

"auia enligido bispo nouarnẽte" $(412, \mathrm{~A}, 40)$ [SV].

porque (de por $+q u e<q u i a)$ : ant. (conj. final): para que.

"tornados em lingoagem por que o entendesem muytos" (75, $A, 75)$ [VT]; "E arrainha dona tareija trabalhaua $\mathrm{E}$ o conde dom fernando por que o fosse“ $(76, \mathrm{~A}, 31)$ [VT].

preito (< placitu-, pelo prov. plait; cf. DP, s.v.), ant. no sent. de: pleito. "nom andemos em este preito" $(29, \mathrm{~B}, 64)$ [CB4]. 
pressa (< pressa, de premere), ant. no sent. de: aflição, dificuldade. "quandoo uy em tall pressa" $(27, \mathrm{~B}, 20)$ [CB3]; "pressas e cuitas e pessares auiã " $(410, \mathrm{~A}, 15)[\mathrm{SV}]$.

vas o, ant. $(<$ vasu- $)$ : copo.

"vasos de vinho" $(24, \mathrm{~B}, 44)[\mathrm{CB} 1]$.

vers o, ant. (< versu-): versículo.

$\mathrm{Na}$ citação a forma sofreu metátese gráfica.

"aquele vesro [sic] / / Oo deus sehor [sic] em mỹ som os teus votos que darom louuores a ti“ $(77, \mathrm{~B}, 9)[\mathrm{VT}]$.

vigília: ant. (<vigilia-): ofício de defuntos.

"fezerom nobres vigilias por ele" $(78, \mathrm{~A}, 16)$ [VT]; “iiij ${ }^{\text {to }}$ kalendas Julj que foy vigilia de sam pedro E de sam paulo" $(76, \mathrm{~B}, 40)$ [VT].

Terminamos pelos termos antigos e/ou populares do vocabulário comum das crónicas estudadas:

a cá, ant. e pop. (de $a$ e cá <ecc(u) hac, tornado *ecc'hac e *eccá; cf. J.P.M., $D E$, s.v. $C a ̂$ ): aqui, cá.

"filharom lhe aqua toda aterra" $(29, \mathrm{~B}, 45)$ [CB4].

a c a e cer, ant. (< accadescěre): acontecer; a c a ec eu : aconteceu. "Acaeceu huũ dia" $(409, \mathrm{~B}, 59)$ [SV].

a l, pronome ant. e popular (lat. vulg. ale- $<$ ălid, ant. neutro; cf. J.P.M., $D E$, s.v. $\left.A l^{\prime}\right)$ : outra coisa; o mais.

"todo oall" $(26, A, 64)$ [CB3].

a lá, ant. e popular (de $a+l a ́<$ illac): lá.

"foi alla obispo" (31, B, 41) [CB4].

a Ig ũ a, ant. ( $<*$ ăliqu'una-, "alicūna-), pl. -s: alguma, -s. "quando algũa ouuesẽ" $(23, \mathrm{~B}, 11)$ [CAN]; "algũa ouelha" $(75, \mathrm{~B}, 72)$ [VT]; "algũas doaçõoes" (22,A, 23) [CAN]; "algũas Reliqujas" (77, A, 42) [VT]; "algũas gemtes" $(420, \mathrm{~A}, 7)[\mathrm{CCA}]$.

a lic ece, ant. (<ár. al-içāç "base, fundamento"; cf. J.P.M., DE, s.v. Alicerce): alicerce.

“no alicece da igreja" (409, A, 19, no título do cap. III) [SV]. 


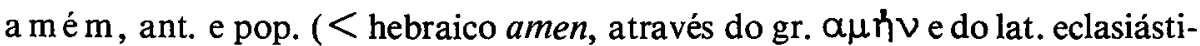
co amen; cf. J.P.M., $D E$, s.v.):

"graças a deus pera senpre amem" $(78, \mathrm{~B}, 63)[\mathrm{VT}]$.

antre, ant. ( $<$ inter): entre.

"antre os quaaes" (29, A, 34) [CB3]; "amtre eles" (417, A, 17) [CCA].

a que ste, -a, -e s, -a s, ant. $\left(<{ }^{*} a c c(u)\right.$ iste, $-a$; - ; -as): este, -a, -es, -as.

O plural já foi formado em português.

"Aqueste cardeall" (30, A, 65) [CB4]; "aquesta mjnha molher" (27, B, 25) [CB3]; "aquestas mããõs" $(27, \mathrm{~B}, 23)$ [CB3].

a s in ha, ant. (<agına-; cf. J.P.M., $D E$, s.v.): depressa.

"majs azinha o podia ganhar" $(416, \mathrm{~A}, 53)$ [CCA1.

a s se s sego, ant. (de assessegar, ant., a prostético + *sessicāre; cf. J.P.M., $D E, 1977, \mathrm{~A})$.

"uiueremos ia ẽ assesego" $(411, \mathrm{~B}, 11)$ [SV].

a tá, ant. (cruzamento de ata < ár. hatta com até < ad *tenes): até. "ataa esta presente Era" (22, A, 19) [CAN]; "(ata) que sse (a)colherom" $(419, \mathrm{~B}, 24)$ [CCA].

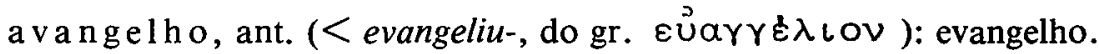
"no auamgelho esta escripto" (78, A, 58) [VT]; "que diz Em no auãgelho" (78, A, 61) $[\mathrm{VT}]$.

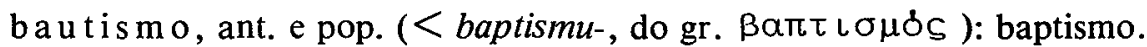
"sancto bautismo" (407, A, 35) [SV].

ca, ant. e pop.: 1 . conj. causal $(<$ quia): porque; 2 . conj. comparativa $(<$ quam): que.

1. " $C a$ assy o diz a cronjca" $(25, \mathrm{~A}, 4)[\mathrm{CB} 1]$; "qua nom avya rrazom" $(27, \mathrm{~B}, 30)$ [CB3]; 2. "semais poder canos" $(29, \mathrm{~B}, 66)$ [CB4]; "mjlhor poder teendes caelle" (29, $B, 69)$ [CB4].

c a õ , ant. (< canu-): de cabelo branco. "mujtos velhos caaos [sic]" (78, A, 25) [VT].

chanto, ant. ( $<$ planctu-): pranto.

"fazemdo grande chanto por dom tello" $(78, \mathrm{~A}, 26)$ [VT].

color, ant. (< cŏlöre-): cor.

Palavra erudita (latinismo).

"negro de sua collor" $(28, \mathrm{~A}, 11)$ [CAN]. 
com peça r, ant. e pop. (cruzamento de empeçar < *impeditiare e *omençar $<{ }^{*}$ cominitiare; cf. Tilander, Notas, ob. cit. na Bibl., p. 7): começar; 1. c o m peça : começa; 2 . com peçou : começou; 3. compeçárom, des. arc.: com(p)eçaram.

1. "Aqui se compeça a estorea" (29, B, 7) [CB4]; 2. "conpeçou a Reinar" $(32, \mathrm{~A}, 3)$ [CB4]; 3. "quando acõpeçarõ “ $(31, \mathrm{~A}, 5)$ [CB4].

compeço, ant. e pop. (der. regressivo de compeçar; v.): começo.

"foy muy boo cristãão Nocom peço" $(31, \mathrm{~B}, 22)$ [CB4].

com prid a m e n te, ant. (de comprido, part. de comprir, arc.): completamente, perfeitamente.

"deus quis fazer compridamẽte mercee" $(408, \mathrm{~B}, 17)$ [SV]. rando.

1. consirar, ant. (< considerare): considerar; 2 . consir a ndo : conside1. "auêmos de consirar" (75, B, 19) [VT].; 2. "consyrando o grande pecado" (77, A, 61) [VT].

corenta, ant. e pop. $(<$ quădrāginta): quarenta.

"quorenta Eciquo" (32, B, 4) [CB4].

corrença, ant. e pop. (de correr): diarreia.

"morrera de corremça" (77, A, 58) [VT].

cu i ta, ant. (de cuitar < * cōctāre; cf. L.V., TA, p. 172, A, s.v. coita): coita.

Para a coexistência de f. com oi e com ui, cf. Huber, Altport., $\$ 44,5$.

"cuitas e pessares auiã “ $(410, \mathrm{~A}, 15)[\mathrm{SV}]$.

delo, ant. e pop. (de dês +lo): dês (ant.) o, desde o.

"delo começo" (414, A, 19) [SV].

dereito, ant. e pop. $(<$ directu-): direito.

"manteedor de dereito" (407, B, 26) [SV]; "linhagem dereita" (25, A, 60) [CB2].

dês, ant. e pop. $(<$ de ex): desde.

“des Ocomeço do Conde Dom anrriqui ataa esta presente Era" (22, A, 18) [CAN];

"desque todo foy guisado" $(26, \mathrm{~A}, 68)$ [CB3].

despois, ant. e pop. ( $<$ de ex poste; cf. J.P.M., $D E$, p. 757, B): depois. "despoys elRey Jsmar abuzy tomou o dicto castello" $(25, \mathrm{~B}, 44)$ [CB2].

despós, ant. (< de ex post; cf. J.P.M., DE, p. 757, B): após, depois (de). "que veesem despos eles" (77, A, 6) [VT]. 
desto, ant. (de de +esto): disto.

"ante desto" (29, A, 53) [CB3]; "Depois desto" (410, B, 5) [SV]; "nom sabendo ho Mestre desto parte" (417, A, 42) [CCA].

d e va çã o, ant. (< devotiōne-): devoção.

"reveremçia e deuação" $(418, \mathrm{~B}, 16)$ [CCA].

din a me nt e, ant. e pop. (de dina, f. de dino + mente): dignamente.

"poderia declarar dinamente" (77, B, 48) [VT].

dino, ant. e pop. $(<$ dignu-): digno.

"muj dina" (75, B, 23) [VT].

(dizer) dezia, ant. e pop. $(<$ dice(b)at): dizia.

"seu padre se dezia odorio" $(75, \mathrm{~B}, 36)$ [VT].

dũ a , ant. e pop. (de $d e+\tilde{u} a)$ : duma.

"natural dhũa uila a que dizem bõna" $(410, \mathrm{~A}, 3)$ [SV].

enteiro, ant. e pop. $(<$ integru-): inteiro.

"o capitoleiro Em teyro" (78, B, 56) [VT].

e n te ir a mente, ant. e pop. (de enteira + mente): inteiramente.

"posoyo em teyramente o senhoryo" $(25, \mathrm{~B}, 35)$ [CB2].

ent once, ant. e brasil. ( $<*$ intŭnce; cf. M.-L., $R E W, \mathrm{n}^{\circ}$ 4518): entonces (ant.), então. V. est once(s).

"foisse entonçe peraassee" $(30, \mathrm{~A}, 46)$ [CB4].

e n tonces, ant. (v. en tonce); o -s é analógico.): então.

"tornousse êtonçes com ell" (30, A, 7) [CB4].

e nve ja, ant. e pop. ( $<$ invidia-): inveja.

"ouueronlhe enueia" (408, A, 22) [SV].

enve jos o, ant. e pop. ( $<$ invidiōsu-): invejoso.

"prazer dos Emvejossos" (78, A, 27) [VT].

esc ontra, ant. e pop.: contra, do lado de.

"hũa porta escusa que estaa (es)contra a Mourarja" (418, A, 55) [CCA].

escuita, f. ant. (de escuitar, ant., < auscultare): escuta, m., esculca. "as suas escujtas que vinhom diante" $(417, \mathrm{~A}, 2)$ [CCA]. 
es prital, ant.: hospital.

"ao espritall de Jerusallem" (29, A, 70) [CB3]; "Prior (do sprital)" (419, B, 8) [CCA].

es prito, ant. e pop. ( $<$ spiritu-): espírito.

"soube pollo esprito santo" $(31, \mathrm{~A}, 3)$ [CB4].

e s to, ant. $(<$ istud): isto.

"Esto nõ he apocrifo" $(25, \mathrm{~A}, 56)[\mathrm{CB} 1]$; "esto fizerom" $(416, \mathrm{~B}, 29)$ [CCA].

e x e m p ro, ant.: exemplo.

"exenpro de bem viuer" $(75, \mathrm{~B}, 28)$ [VT].

(fazer) fezeste, ant. e pop. (< fëcisti): fizeste; f ezestes, ant. e pop. $(<$ fécistis): fizestes; f e ze r a , ant. e pop. (<fëcĕrat, com deslocação do acento): fizera; f e zesse, ant. e pop. (<fécŭsset): fizesse; fezess e m, ant. e pop. $(<$ fécissent): fizessem; f e z e r e m , ant. e pop. ( $<$ fecerint, com deslocação do acento): fizerem.

"esto que tu fezeste" $(412, \mathrm{~A}, 29)$ [SV] ; "este bispo que ffezestes" $(28, \mathrm{~A}, 33)$ [CB3]; "que dom egas monjz fezera" (27, B, 2) [CB3]; "que fezese as lex" (25, B, 39) [CB2]; "da Jnliçõ quefezesem das abadesas" $(23, \mathrm{~B}, 10)$ [CAN]; "se algũas pessoas fezeren bẽ $(413, \mathrm{~B}, 62)[\mathrm{SV}]$.

(ficar), ant. no sentido de: fincar, fi cá r o m, des. arc.: ficaram. "ficarõ [perf.] os geolhos ẽ terra" $(409, \mathrm{~A}, 16)$ [SV].

f i ú za, ant. e pop. ( $<$ fidūcia-): confiança.

"com muj grande fiuza" $(77, \mathrm{~B}, 45)$ [VT].

f r a mengo, ant. ( $<$ germ. flaming; cf. $D P$, s.v. Flamengo): flamengo. "auia nome galtero e era framẽgo" $(413, \mathrm{~A}, 51)$ [SV].

(gastar) ga stada, ant. com o aux. ser: gasta.

“De(s)pojs que a noyte foy gastada" $(417, \mathrm{~A}, 9)$ [CCA].

h om e, ant. e pop. ( $<$ hominë-): homem.

"boos homes" (75, B, 16) [VT].; "dous homes boos" $(407, \mathrm{~B}, 31)$ [SV].

i migo, ant. ( $<$ inimīcu-): inimigo.

"algũũs emijgus" (76, A, 40) [VT].

ind i no, ant. e pop. ( $<$ indignu-): indigno.

"O prior de santa cruz Jndino" (78, B, 3) [VT]. 
1. la ix a r, ant. (< laxāre): deixar; 2. le ix o, ant. (< laxo): deixo; 3. le ixav a, ant. (<laxăbat); 4. le i xá v o m, ant., des. arc. e 5. lei x a v a m, ant. (<laxăbant): deixavam; 6. le ix o u, ant. (< laxaut, por laxävit): deixou; 7. le ix á m os, ant. (< laxā(vĭ)mus): deixámos; 8. le ixá rom, ant., des. arc. (<laxāa(ve)runt): leixaram, ant., deixaram; 9. le ix a r a, ant. (<laxa(ve)rat): deixara; 10. le ix a r emos, ant. (<*laxar'emus): deixaremos; 11. leix a redes, ant., des. arc. $(<$ *liexar'êtis): leixareis, ant, deixareis; 12. le ix res, ant. (<laxa(ve)ris): deixares; 13. I i ixes, imperati. (neg.), ant. (< laxes): deixes;14. le ix a da , ant. (<laxäta-): deixada.

1. "leixar ẽ el dos seus bẽẽs" $(413, \mathrm{~B}, 59)$ [SV].; "leixar a Tore" $(416, \mathrm{~B}, 20)$ [CCA]; 2. "que eu leixo" $(26, \mathrm{~A}, 46)$ [CB3]; 3. "leixaua de fazer" $(416, \mathrm{~A}, 50)$ [CCA]; 4. "não leixavom os moros de ho combater" $(417, \mathrm{~B}, 2)$ [CCA]; 5."leixauã ao dito Moesteiro grãdes partes de seus algos" (413, B, 43) [SV].; 6. "leyxou o cargo" (24, $\mathrm{B}, 37)$ [CB1]. "leixamos [perf.] de escripuer" (78, B, 43) [VT]; 8. "leixarom [perf.] o trassundo" (77, A, 53) [VT]; 9. "leixara o dito Moesteiro" $(414, \mathrm{~B}, 12)$ [SV]; 10. "leixaremos aquy afallar" $(27, \mathrm{~B}, 37)$ [CB3]; 11. "leixaredes aterra" $(29, \mathrm{~B}, 65)$ [CB4]; 12. "o dia que a leixares deffazer" $(26, A, 55)$ [CB3]; 13. "nõ leixes de ffazer" (26, $\mathrm{A}, 53)$ [CB3]; 14. "fora leixada per hũũ homẽ" $(411, \mathrm{~A}, 3)$ [SV].

log o, ant. $(<\operatorname{locu}$-): lugar.

"asee desse $\log O^{\text {" }}(24, \mathrm{~B}, 40)$ [CB1]; "tijnha sempre na dita cidade logo iulgauil por elRey“ (407, B, 24) [SV].

$\mathrm{mi}$, ant. e pop. $(<(m \bar{i}$, por $m \bar{l} h \bar{\imath}): \mathrm{mim}$.

"cõ todo seu dotamẽto fique a $m i$ " $(412, \mathrm{~B}, 21)$ [SV].

milhor, ant. e pop. ( $<$ mĕliōre-): melhor.

$\mathrm{O} i$ deve-se a infl. da palatal seguinte.

"omilhor homem darmas" (26, A, 32) [CB3].

(mover): sent. ant.: mover-se, partir; movêrom, des. arc. $(<-\breve{u n t})$ : moveram.

"moverom [perf.] todos daly" $(417, \mathrm{~A}, 12)[\mathrm{CCA}]$.

ne miga lha, ant. (< nec+ *micalea-; cf. J.P.M., $D E$, p. 1503, A): nada. "nom teuese de veer hy nemjgalha" $(77, \mathrm{~A}, 12)$ [VT].

nom, ant. ( $<$ non): não.

"aescriptura que nom for uerdadeira" $(22, \mathrm{~A}, 34)$ [CAN]; "que lha nom cortasse" $(28, \mathrm{~A}, 38)$ [CB3].

ò s, ant. e pop. (contracção de aos < ad illos): aos.

"preguntou affomso Enriquez os uassalos" (29, B, 41) [CB4].

ou trossi, ant. (de outro $<$ altërŭ- $+s i<$ sic): outrossim. "Outrosy uençeeo hũã mujto grande batalha" $(25, \mathrm{~B}, 62)$ [CB2]. 
pendença, ant. ( $<$ poenitentia-): penitência.

"lugar de peendença" $(76, \mathrm{~B}, 62)$ [VT]; "grande pendemça em seruiço de deus" (77, $\mathrm{B}, 11)[\mathrm{VT}]$.

pera, ant. $(<$ per $+a d)$ : para.

"pera tirar estas duuidas" (22, A, 28) [CAN]; "pera atanto bem" $(76, \mathrm{~A}, 42)$ [VT].

perca lça r, ant. (< * percalceāre; cf. J.P.M., DE, p. 462, B): alcançar. "as coussas que eu aqui podesse percalçar" $(411, \mathrm{~B}, 36)$ [SV].

pero (ou peró), ant. (< per hoc): 1. embora; 2 . em-que: se bem que. 1. "pero tamto tempo Senhorezasse" $(25, \mathrm{~B}, 37)[\mathrm{CB} 2]$; 2. "em pero que era moço" $(76, \mathrm{~A}, 36)[\mathrm{VT}]$.

pla n to, ant. (< planctu-): pranto.

"o palnto (sic) E choro" (78, A, 17) [VT].

1. poer, ant. (<pōnĕre): pôr; 2. poermos, ant. (<poner(e)mus): pormos; 3. poer em, ant. (< ponĕrent, com deslocação do acento): porem; 4 . p o e re mos, ant. (<poner'emus): poremos.

"mandou poer çeuada" (28, A, 62) [CB3]; 2. "e a poermos ẽ poder de cristaãos" $(413, \mathrm{~B}, 50)$ [SV]; 3. "pera lhes poerem o fogo" $(419, \mathrm{~B}, 40)$ [CCA]; 4. "o poeremos" $(413, \mathrm{~B}, 8)[\mathrm{SV}]$.

polo, -a, -os, -a s, ant. e pop. (de por +lo, etc.): pelo, -a, os, as. "pollo ffazer alleuantar" (27, A, 36) [CB3]; "polas almas" $(409, \mathrm{~B}, 11)$ [SV]; "polo seu sancto acordo" (407, B, 32) [SV]; "pola uida" (409, A, 2) [SV].; "pollos peccadores saluar" $(24, A, 17)[C B 1]$.

(povorar), ant. (< populare): povoar; 1. povorou, ant.: povoou; 2. povor a d a , ant.: povoada.

1. "elle pouorou coujlhaa" $(26, \mathrm{~A}, 1)$ [CB2]; 2. "era majs pouorada" $(416, \mathrm{~B}, 28)$ [CCA].

(prazer); 1. prougue, ant. ( $<$ placuit $)$ prouve; 2 . prouguesse, ant. $(<$ placuisset $)$ : prouesse.

1. "prouguelhe muyto" (413, A, 55) ]SV]; 2. "prouguese a deus" (76, B, 50) [VT].

(preçar), ant. (< pretiäre): prezar; preçavam, ant. (<pretiäbant): prezavam.

“preçauã e onrrauã cada uez mais o dito Mosteiro" (413, A, 25) [SV].

p rol, ant. (sing. deduzido do plur. proes, por anal. com sol - soes; cf. D. C.M., Gloss. do CA, s.v.): proveito; servir a - : aproveitar, ser útil.

"nom entendo hi auossa proll" $(27, \mathrm{~A}, 70)$ [CB3]; "A qual rrenenbrança serue a proll“ $(22, \mathrm{~A}, 22)[\mathrm{CAN}]$. 
p rove, ant. e pop. (< lat. vulg. *pōpĕre, através das formas pobre e *povre; cf. Neto, Fontes, p. 21, v. Bibliog.): pobre.

"que a desse a proues" $(411, \mathrm{~A}, 5)$ [SV].

(repairar), ant. (< rĕpariāre); cf. Du Cange (ob. cit. na Bibl.), s.v., repariare ve$l a=$ renavigare, em Solinus, gramático da $1^{\text {a }}$ metade do séc. III d.C.; "retro dare cursum, ut habet Hofmannus in Lexico.“): reparar; repairou, ant. (< *repariaut, por reparavit): reparou.

"rrepairou todas as ffortellez" $(29, \mathrm{~A}, 25)$ [CB3].

rezã o, ant. e pop. (< rătiōne-): razão.

"por esta rezão" (419, B, 41) [CCA].

r om e u, ant. (do prov. romeu; cf. J.P.M., $D E$, p. 1908, B): romeiro.

"os romeus que uam ẽ ierusalem" $(413, \mathrm{~A}, 16)$ [SV].

s a, ant. (< sua-, por próclise): sua (pl.: s a s $(<$ suas, id.): suas.

"toda ssa terra" (27, B, 44) [CB3]; "cõ ssas perteeças" (29, B, 17) [CB4].

s a lu ço, ant. e pop. (< sügglüttıu-; cf. J.P.M., DE, p. 2001, A): soluço.

Houve dissimilação $(o-\mathrm{u}>\mathrm{a}-\mathrm{u})$.

"oo que saluços tam grandes daua" $(77, \mathrm{~B}, 15)[\mathrm{VT}]$.

(ser): 1. s om, ant. (<sŭm): sou; 2. so des, ant. (< sŭtis): sois.

1. "como eu soom" $(26, \mathrm{~B}, 49)$ [CB3]; 2. "ssodes mj̃zcrado" $(28, \mathrm{~A}, 32)$ [CB3].

s e n hos, ant. $(<$ singŭlos): numeral distributivo.

"acadahũũ senhos pããẽs" $(24, \mathrm{~B}, 44)$ [CB1].

s impres, ant. (< simplice-): simples.

A ortogr. actual grafa com $-s$, por convenção, visto a pal. ser grave.

"estas çinprez palauras" (78, A, 67) [VT].

s ô, ant. $(<s u b)$ : sob.

"foy so a era demlj e cento e setẽẽta annos" $(25, \mathrm{~A}, 24)$ [CB1].

súd i to, ant. (< subditu-): súbdito.

"as võõtades dos suditos" $(75, \mathrm{~B}, 69)$ [VT].

sú pi to, ant. e pop. $(<$ subĭtu-): súbito.

O $p$ deve-se a ensurdecimento; cf. Viana, Apostilas, t. II, p. 448.

"derom de supito nos Christãos" (417, A, 30) [CCA].

(ta), ant.; pl.: tas (<tuas, por próclise): tua, tuas.

"quebrantadas sejã as tas pernas" $(30, \mathrm{~A}, 18)$ [CB4]. 
(teer); 1. tevera, ant.e pop.: tivera; 2 . tevesse, ant. e pop.: tivesse. 1. "nunqua tevera tempo" $(25, \mathrm{~B}, 41)$ [CB2]; 2 . "tevesse bispo e see" $(412, \mathrm{~A}, 18)$, no tít. do cap. XI) [SV].

tôdolos, -alas, ant. (<*tōtōs-los, *tōtās-las; cf. E.W., From Latin, $\S 109,3)$ : todos, -as.

"todollos Reys" (25, B, 30) [CB2]; "todolos christaãos" (409, A, 23) [SV]; "todallas ffortellezas“ (26, B, 65) [CB3].

1. t r a ge r, ant. (<*trăgĕre, por trăhĕre; cf. J.P.M., DE, s.v.trazer): trazer; 2. tragem, ant. (<*tragent, por *tragunt): trazem; 3 . tragia, ant. $(<$ *tragè (b)at): trazia; 4. t r a g i a m, ant. $(<*$ tragè(b)ant): traziam; 5 . t r o u ve, ant. e pop. (por analog. com houve): trouxe; 6. trouvérom, ant. e pop. (por. anal. com houvérom): trouxeram; 7. tr a ge, ant. ( $<*$ trage): traz(e); 8. tragendo, ant. $(<*$ tragendo): trazendo.

1. "pera elrey trager os pobos" $(412, \mathrm{~B}, 2)$ [SV]; 2. "que tragẽ os romeus" $(413, \mathrm{~A}$, 16) $[\mathrm{SV}]$; 3. "tragia suas gentes" $(408, \mathrm{~A}, 8)$ [SV]; 4. "offertas que tragiam" $(409, \mathrm{~B}$, 14) $[\mathrm{SV}]$; 5. "trouue demarocos os marteres" $(31, \mathrm{~A}, 57)$ [CB4]; 6. "trouueromnos a villa" $(418, \mathrm{~B}, 7)$ [CCA]; 7. "trageo [imperat.] aqui" $(410, \mathrm{~B}, 21)$ [SV]; 8. "ẽ lhes tragendo" $(408, \mathrm{~B}, 18)[\mathrm{SV}]$.

u, ant. ( $<\breve{u} b i$, por metafonia e próclise; cf. L.V., TA, p. 194, B): onde. "per hu ell uem" (28, A, 35) [CB3].

ũ a, ant. e pop. $(<u n a)$ : uma.

"hũa dona" (22, B, 27) [CAN]; "hũa cruz de prata" (24, B, 60) [CB1]; "ouueram hũa filha" (25, A, 49) [CB1]; "huũa grande parte" $(29, \mathrm{~B}, 12)$ [CB4]; "hũũ̃ dona" (31, B, 5) [CB4]; "hũa grande enfirmjdade“ (76, A, 27) [VT]; "hũa pedra" (409, A, 31) [SV]; “hũa forte peleja“ $(417, \mathrm{~A}, 17)$ [CCA].

vig a i r o, ant. e pop. ( < vicariu-): vigário. “uigayro geeral" $(408, \mathrm{~B}, 57)[\mathrm{SV}]$. 


\section{BIBLIOGRAFIA}

mais importante

Costa (J. Almeida) e A. Sampaio e Melo, Dicionário da Língua Portuguesa. $5^{\text {a }}$ edição. Porto, s.d.

Du Cange, Glossarium Mediae et Infimae Latinitatis. Niort, 1883-1887.

Hubert (Joseph), Altportugiesisches Elementarbuch. Heidelberga, 1933.

Lapa (M. Rodrigues), Lições de Literatura Portuguesa/Época Medieval. Lisboa, 1934 (e todas as edições posteriores, nomeadamente a quinta, de 1964).

Machado (José Pedro), Dicionário Etimológico da Língua Portuguesa. $1^{\text {a }}$ edição. Lisboa, 1952-1959.

Influência Arábica no Vocabulário Português. 2 vols. Lisboa, 1958-1961.

Meyer-Lübke (W.), Romanisches Etymologisches Wörterbuch. $3^{a}$ edição. Heidelberga, 1935.

Nascentes (Antenor), Dicionário Etimológico da Língua Portuguesa. 2 tomos. Primeira Edição. Rio de Janeiro, 1932 e 1952.

Silva Neto (Serafim da), Fontes do Latim Vulgar. $3^{\text {a }}$ edição. Rio de Janeiro, 1956.

Tilander (Gunnar), Notas Etimológicas/Separata del Homenaje a Dámaso Alonso/ Tomo III. Madrid, 1963.

Vasconcelos (Carolina Michaëlis de), Glossário do Cancioneiro da Ajuda, in Revista Lusitana, vol. XXIII, até p. 95. Lisboa, 1920.

Vasconcelos (José Leite de), Antroponímia Portuguesa. Lisboa, 1982.

Lições de Filologia Portuguesa. $2^{\mathrm{a}}$ edição. Lisboa, 1926.

Opúsculos. Volume III: Onomatologia. Coimbra, 1931.

Textos Arcaicos. $4^{\mathrm{a}}$ edição. Lisboa, 1959.

Viana (Gonçalves), Apostilas aos Dicionários Portugueses. 2 tomos. Lisboa, 1906.

Williams (Edwin B.), From Latin to Portuguese/Historical Phonology and Morphology of the Portuguese Language. Second Edition. Filadélfia, 1962.

\section{Povzetek}

\section{STARE BESEDE V PORTUGALSKIH SREDNJEVEŠKIH KRONIKAH}

Jezik stare portugalščne, kot ga razkrivajo srednjeveške kronike, zbrane v Portugaliae Monumenta Historica, daje veliko gradiva za študij etimologije in semantike. Pojavljajo se lastna imena, ki so latinskega in tudi grškega izvora, zapisana pa so tudi germanska in arabska. Enako velja za splošno besedišče, kjer pa je seveda latinski fond daleč najpomembnejši. Za semantiko je dragocena vrsta izrazov, ki imajo $v$ današnji portugalščini arhaičen pridih. Posebne pozornosti so vredni tudi tisti izrazi, ki so danes sicer v ljudski rabi, imajo pa v starih kronikah svojske, danes ne več poznane pomene. 